\title{
Short-Term Effects of Repetitive Transcranial Magnetic Stimulation on Speech and Voice in Individuals with Parkinson's Disease
}

\author{
L. Hartelius ${ }^{a} \quad$ P. Svantesson ${ }^{a} \quad$ A. Hedlund ${ }^{a} \quad$ B. Holmberg ${ }^{b} \quad$ D. Revesz ${ }^{b}$ \\ T. Thorlin ${ }^{b}$
}

Divisions of a Speech and Language Pathology and ${ }^{\mathrm{b}}$ Neurology, Institute of Neuroscience and Physiology, Sahlgrenska Academy at the University of Gothenburg, Gothenburg, Sweden

\section{Key Words}

rTMS $\cdot$ Parkinson's disease $\cdot$ Voice $\cdot$ Speech $\cdot$ Acoustic analysis

\begin{abstract}
The main characteristics of dysarthria in Parkinson's disease (PD) are monotony of pitch and loudness, reduced stress, variable speech rate, imprecise consonants, and breathy and harsh voice. Earlier treatment studies have shown that dysarthria is less responsive to both pharmacological and surgical treatments than other gross motor symptoms. Recent findings have suggested that repetitive transcranial magnetic stimulation (rTMS) may have a beneficial effect on vocal function in PD. In the present study, 10 individuals with mild PD and no or minimal dysarthria were treated with rTMS as well as placebo stimulation in a blinded experiment. Stimulation was delivered using a frequency of $10 \mathrm{~Hz}$ and a stimulation intensity of $90 \%$ of the motor threshold. The site of stimulation was the cortical area corresponding to the hand, on the hemisphere contralateral to the patient's most affected side. The participants were audio-recorded before and after both rTMS and sham stimulation. Acoustic analysis was performed on 3 sustained /a:/ for each of the 4 conditions, and analyzed both for the whole group as well as for men and women separately. Results showed that there were no
\end{abstract}

significant differences between any of the conditions regarding duration of sustained fricative or sustained vowel phonation, diadochokinetic rates or intelligibility. Above all, the results of acoustic analyses showed an effect of placebo; there was a significant reduction in fundamental frequency $\left(F_{0}\right)$ variation, pitch period perturbation, amplitude period perturbation, noise-to-harmonics ratio and coefficient of variation in $\mathrm{F}_{0}$ between the recordings performed before compared to after sham stimulation.

Copyright $\odot 2010$ S. Karger AG, Base

\section{Introduction}

Parkinson's disease (PD) is caused by basal ganglia dysfunction and creates a movement disorder characterized by bradykinesia (slow movements), muscle rigidity and resting tremor. One of the most frequent and disabling symptoms is dysarthria, a neurological motor speech impairment that is characterized by slow, weak, imprecise, and/or uncoordinated movements of the speech musculature involved in respiration, phonation and articulation. Dysarthria in PD is defined as hypokinetic, and occurs in about $90 \%$ of people with autopsy-confirmed PD [1]. The main audible symptoms are monotony of pitch and loudness, reduced stress, variable speech rate, imprecise con-

\section{KARGER}

Fax +4161306 1234

E-Mail karger@karger.ch

www.karger.com (c) 2010 S. Karger AG, Basel

1021-7762/10/0623-0104\$26.00/0

Accessible online at:

www.karger.com/fpl
Lena Hartelius, PhD, Division of Speech and Language Pathology

Institute of Neuroscience and Physiology

Sahlgrenska Academy at the University of Gothenburg

Box 452, SE-405 30 Gothenburg (Sweden)

Tel.+46 31786 6884, E-Mail lena.hartelius@neuro.gu.se 
sonants, and a breathy and harsh voice [2]. Dysarthria can cause a significant reduction in speech intelligibility, i.e. a person with moderate-severe dysarthria has considerable difficulties making himself/herself understood to listeners. Intelligibility was found to be below the control mean of unaffected speakers in $<70 \%$ of 125 persons with PD in a study by Miller et al. [3].

To characterize the speech disorder in individuals with PD, a number of measures have been used. Clinical characterization includes oral motor and speech examination and perceptual assessment of different types of speech samples, e.g. sustained phonation, syllable repetitions (diadochokinetic rates), text reading and spontaneous speech [4]. In addition, acoustic analysis can provide a more objective and reliable means to index the severity and characteristics of dysarthria in general and hypokinetic dysarthria in particular [5]. Analysis of sustained phonation has been shown to be a sensitive measure of subclinical symptoms, and is a potential biomarker of early disease progression and treatment [6-8].

It is well known that pharmacological and surgical interventions, although successful in decreasing global motor limb dysfunction, are minimally effective in reducing speech and voice symptoms. Dopamine therapy has been reported to improve speech functioning, but group studies show great variability in results and recent reviews indicate that the majority of studies have failed to find a causal relationship between levodopa and functional speech intelligibility in individuals with PD. Moreover, there is evidence to suggest other etiologies for the speech problems, such as deficits in internal cueing, scaling movement force and amplitude, sensorimotor gating, selfperception of voice, etc. $[9,10]$. Among surgical techniques, deep brain stimulation of the subthalamic nucleus has been shown to yield dramatic improvement in global motor function of the limbs and to reduce tremor, but its effects on speech are varied and inconclusive. Dysarthric symptoms frequently appear as a side effect and a preexisting dysarthria can be worsened. Dysarthria is reported as an adverse side effect in up to $14 \%$ of patients. Recent findings suggest that other simulation sites, such as the caudal zona incerta or pedunculopontine nucleus, might be more promising in terms of speech effects [11, 12].

Compared to deep brain stimulation, the use of noninvasive brain stimulation has significant advantages, such as not involving surgical procedures and having relatively mild adverse effects. Transcranial magnetic stimulation (TMS) was introduced by Barker et al. [13] in 1985, as a means of studying the central nervous system. Repetitive pulses (rTMS) can be used to modulate the excit-

Effects of rTMS on Speech and Voice in $\mathrm{PD}$ ability of the brain area targeted, and this has been studied in many neurological and psychiatric patient populations. rTMS can either disrupt neural activity and interfere with cortical functioning or enhance motor cortex excitability and facilitate cortical functioning, depending on frequency of stimulation. A recent systematic review of the effects of rTMS on motor signs in PD [14] included 10 randomized controlled clinical trials and used the motor section (part III) of the Unified Parkinson's Disease Rating Scale (UPDRS) as outcome. The meta-analysis yielded an effect size of -0.58 in UPDRS for high-frequency $(>1 \mathrm{~Hz})$ rTMS studies and no significant effects for low-frequency $(<1$ $\mathrm{Hz}$ ) rTMS studies. There were 152 patients in the highfrequency group and 123 patients in the low-frequency group. It was concluded that high-frequency rTMS can modulate underactive brain regions in individuals with PD and produce clinically significant motor improvement. All included trials showed this reduction. So far, to our knowledge, only 1 published study has described the effects of rTMS on voice and speech in PD [15]. In this study, 30 patients were given active or sham $15-\mathrm{Hz}$ rTMS to the left dorsolateral prefrontal cortex in a first experimental session and active 5-Hz rTMS of the primary motor cortex mouth area in a session study. A rater, who was blind to the aim of the study and the condition of the patients, evaluated speech characteristics using perceptual and acoustic measures. Voice-related quality of life was also evaluated. Results showed that rTMS of the left dorsolateral prefrontal cortex resulted in mood amelioration and subjective improvement of the voice-related quality of life, but not in objective measures such as fundamental frequency and voice intensity. However, rTMS of the primary motor cortex mouth area induced significant improvements in the fundamental frequency (i.e. decreased $\mathrm{F}_{0}$ in men and increased $\mathrm{F}_{0}$ in women) and voice intensity.

The aim of the present study was to explore the effects of high-frequency rTMS in a group of individuals with PD. The main focus of interest was the effect on global motor function and hand motor function, and voice and speech was monitored for secondary treatment effects or to document any adverse effects.

\section{Materials and Methods}

\section{Participants}

Participants were recruited from the Movement Disorders Clinic at Sahlgrenska University Hospital by senior neurologists specialized in PD. Ten patients (6 male, 4 women) with early-stage PD aged 39-67 years (mean age \pm SD: $57.0 \pm 8.9$ years) participated in the study. Disease duration was $1-7$ years (mean: $3.6 \pm$ 
2.4 years) and symptoms were mild with average UPDRS III scores of $15.6 \pm 7.6$. The study was approved by the local ethics committee and was performed in accordance with the ethical standards of the Declaration of Helsinki. All patients gave their written informed consent prior to initiation of the study.

\section{Study Design}

A placebo-controlled crossover design was used, in which the participants were unaware of what kind of stimulation was given. They were informed about the rTMS procedure and its potential to provide transitory clinical improvement in parkinsonian motor symptoms. They were also made aware that in one session they were going to receive sham rTMS (placebo-rTMS) and in another active rTMS stimulation, but they did not know which type of stimulation was given on which occasion. All participants came to the Movement Disorders Laboratory for 2 treatment sessions (day 1 and day 2) with 1 week's interval in between. All medication was withheld for $12 \mathrm{~h}$ prior to each session. Sham rTMS was given during the initial session (day 1) in all cases in order to avoid potential long-term effects of real rTMS to the following week.

\section{rTMS Treatment}

Four blocks of 20-train 10-Hz rTMS (train duration: $2.5 \mathrm{~s}$; inter-train intervals: $5 \mathrm{~s}$ ) were applied over the motor hand area contralateral to the more severely affected upper limb. Each 20-pulse train was followed by a 4-min break in order to cool the coil. In total, the participant received 2,000 rTMS pulses during each stimuli session. The motor threshold, which was determined for each individual prior to the rTMS sessions, was defined as the lowest stimulus intensity able to elicit a muscular contraction from the contralateral musculus abductor pollicis brevis. When the area was found, it was marked as a point on the scalp of the patient with an ink pen. The stimulation intensity was set at $90 \%$ of the resting motor threshold for the musculus abductor pollicis brevis. The coil was held in a fixed position by a mechanical arm over the motor cortex and constant coil position was continuously monitored for the duration of the treatment session. The patients were seated comfortably in a chair with armrests and headrest. During both sham and real rTMS, all patients wore ear plugs in order to protect the ear from the noise associated with the discharge of the stimulation coil. Four blocks of rTMS were delivered throughout the day. Biphasic rTMS pulses were delivered through a figure-of-eight coil attached to a MagPro X100 (Medtronic). Sham rTMS was performed with a commercially available figure-of-eight coil (MCF$\mathrm{P}-\mathrm{B} 65$, Medtronic), this sham coil has the appearance of and provides the same noise as a real rTMS coil.

\section{Recorded Samples and Equipment}

Four recordings were made: (1) day 1 before treatment, (2) day 1 after sham rTMS, (3) day 2 before treatment, and (4) day 2 after real rTMS. Participants were off medication during all recordings, which took place in the Movement Disorders Laboratory. Speech samples included maximum fricative duration /s:/ repeated 3 times, sustained vowel phonation /a:/ repeated 3 times, syllable repetitions (alternating and sequential; /papapa.../, /tatata.../, /kakaka.../, /patakapataka.../), intelligibility test sentences [16] and the reading of a standard passage. Participants were recorded using a digital audio tape recorder (Sony Walkman TCD D-7) and a table electric condenser microphone (Sony ECM-MS957) with a constant mouth-to-microphone distance of approximately $30 \mathrm{~cm}$.
Most recordings were done by 2 of the researchers (P.S. and A.H.), although 2 other people were called in to do the recordings on a few occasions. The verbal instructions on how to perform the speech tasks were available in writing and were given identically to all participants on all 4 occasions.

\section{Acoustic Analysis}

The sustained vowel phonations of one participant (\#6) were not recorded due to a technical error. All other sustained vowel phonations ( 4 recording sessions $\times 3$ vowels $=12$ sound files for each of the 9 participants) were digitized using Adobe Audition 2.0 at a sampling rate of $44 \mathrm{kHz}$. Acoustic analysis was performed using the Multi-Dimensional Voice Program (MDVP, Computerized Speech Lab CSL 4400, Kay Elemetrics). Due to the nature of the study, recording conditions were not optimal. The Movement Disorders Laboratory is not a sound-proof environment, and when the recorded vowels were scrutinized in Adobe Audition, there were several occasions where external disturbances were picked up by the microphone and interfered with the participant's voice. Instead of editing the sound files, it was decided that approximately a 3 -second mid-section from each recording for each person under the 4 conditions (i.e. 44 sound files) would be selected for acoustic analysis. The mid-section was chosen to avoid voice onset and offset effects [17]. Nine voice parameters included in MDVP, known to be affected by signs of hypokinetic dysarthria and thus possibly reflecting changes associated with $\mathrm{PD}$, were selected.

\section{Statistical Analysis}

Four comparisons were made: (1) recording 1 versus recording 2 , i.e. effects of sham rTMS, (2) recording 3 versus recording 4, i.e. effects of real rTMS, (3) recording 1 versus recording 3 , i.e. longterm effects of sham rTMS, and (4) recording 2 versus recording 4 , i.e. difference between real rTMS and sham rTMS. Significant differences were determined using t tests with Bonferroni correction $(\mathrm{p}<0.0125)$.

All acoustic analyses were performed by P.S. and A.H. in collaboration. To ensure reliability of measurement, $10 \%$ of the MDVP analyses were also done by an external person for comparison. The reliability analysis included choosing a 3-second portion in the selected vowels and performing the MDVP analysis. There was a strong correlation (Pearson $r=0.99$ ) between the 2 analyses.

\section{Results}

\section{Speech}

Results regarding sustained fricative duration (/s:/, in seconds), sustained vowel phonation (/a:/, in seconds), diadochokinetic rates (syllable repetition /kakaka.../ selected, in syllables per second) and sentence intelligibility for all 10 participants comparing the 4 different recordings are presented in table 1 . No significant differences between any of the conditions were found.

\section{Voice}

The results of acoustic analysis with MDVP are shown in table 2 , and presented as $\mathrm{p}$ values for significant differ- 
Table 1. Results comparing maximum fricative duration and vowel phonation, diadochokinetic rate and sentence intelligibility

\begin{tabular}{|c|c|c|c|c|c|c|c|c|c|c|c|c|c|c|c|c|}
\hline \multirow[t]{2}{*}{$\begin{array}{l}\text { Parti- } \\
\text { cipant }\end{array}$} & \multicolumn{4}{|c|}{$\begin{array}{l}\text { Maximum fricative duration /s:/ } \\
\text { seconds }\end{array}$} & \multicolumn{4}{|c|}{$\begin{array}{l}\text { Sustained vowel phonation /a:/ } \\
\text { seconds }\end{array}$} & \multicolumn{4}{|c|}{$\begin{array}{l}\text { Diadochokinetic rate /kakaka.../ } \\
\text { syllables/second }\end{array}$} & \multicolumn{4}{|c|}{$\begin{array}{l}\text { Sentence intelligibility } \\
\%\end{array}$} \\
\hline & $\mathrm{R} 1$ & $\mathrm{R} 2$ & $\mathrm{R} 3$ & $\mathrm{R} 4$ & $\mathrm{R} 1$ & $\mathrm{R} 2$ & R3 & $\mathrm{R} 4$ & $\mathrm{R} 1$ & R2 & $\mathrm{R} 3$ & $\mathrm{R} 4$ & $\mathrm{R} 1$ & $\mathrm{R} 2$ & R3 & $\mathrm{R} 4$ \\
\hline 2 & 29.20 & 24.00 & 18.43 & 21.60 & 14.87 & 13.40 & 15.83 & 18.03 & 6.70 & 6.70 & 6.40 & 6.70 & 96.15 & 96.15 & 96.15 & 100.00 \\
\hline 3 & 17.07 & 16.37 & 15.67 & 16.27 & 14.03 & 14.67 & 17.67 & 17.17 & 4.40 & 4.80 & 4.00 & 4.20 & 100.00 & 100.00 & 100.00 & 100.00 \\
\hline 4 & 29.27 & 29.90 & 23.37 & 26.83 & 31.80 & 38.20 & 34.87 & 34.60 & 4.00 & 4.10 & 4.30 & 4.30 & 96.15 & 100.00 & 96.15 & 100.00 \\
\hline 7 & 17.20 & 17.73 & 17.50 & 19.43 & 14.33 & 15.57 & 17.43 & 15.83 & 5.50 & 5.80 & 5.60 & 5.25 & 96.15 & 100.00 & 100.00 & 100.00 \\
\hline 8 & 12.90 & 12.20 & 15.30 & 11.73 & 10.03 & 9.87 & 10.20 & 12.53 & 6.40 & 6.20 & 5.80 & 5.80 & 100.00 & 100.00 & 96.15 & 100.00 \\
\hline 9 & 15.17 & 13.37 & 15.20 & 13.90 & 6.10 & 8.47 & 8.17 & 10.57 & 5.10 & 5.60 & 5.20 & 5.20 & 100.00 & 100.00 & 100.00 & 100.00 \\
\hline 10 & 9.87 & 8.07 & 6.67 & 6.40 & 20.07 & 23.17 & 20.77 & 23.43 & 5.45 & 5.70 & 5.30 & 5.70 & 92.31 & 100.00 & 100.00 & 100.00 \\
\hline $\begin{array}{l}\text { Mean } \\
\pm S D\end{array}$ & $\begin{array}{c}16.19 \pm \\
7.55\end{array}$ & $\begin{array}{c}15.86 \pm \\
6.65\end{array}$ & $\begin{array}{c}14.50 \pm \\
4.91\end{array}$ & $\begin{array}{c}14.76 \pm \\
6.28\end{array}$ & $\begin{array}{c}15.24 \pm \\
7.28\end{array}$ & $\begin{array}{c}16.75 \pm \\
9.14\end{array}$ & $\begin{array}{c}16.26 \pm \\
8.17\end{array}$ & $\begin{array}{c}17.54 \pm \\
7.56\end{array}$ & $\begin{array}{l}5.34 \pm \\
0.86\end{array}$ & $\begin{array}{l}5.44 \pm \\
0.79\end{array}$ & $\begin{array}{l}5.32 \pm \\
0.77\end{array}$ & $\begin{array}{l}5.37 \pm \\
0.79\end{array}$ & $\begin{array}{l}97.31 \pm \\
3.17\end{array}$ & $\begin{array}{l}98.85 \pm \\
2.60\end{array}$ & $\begin{array}{l}98.85 \pm \\
1.86\end{array}$ & $\begin{array}{l}99.23 \pm \\
2.43\end{array}$ \\
\hline
\end{tabular}

No differences are statistically significant. R1 = Day 1 before treatment; R2 = day 1 after sham rTMS; R3 = day 2 before treatment; R4 = day 2 after real rTMS.

Table 2. Overview of significant differences in voice quality as measured with MDVP

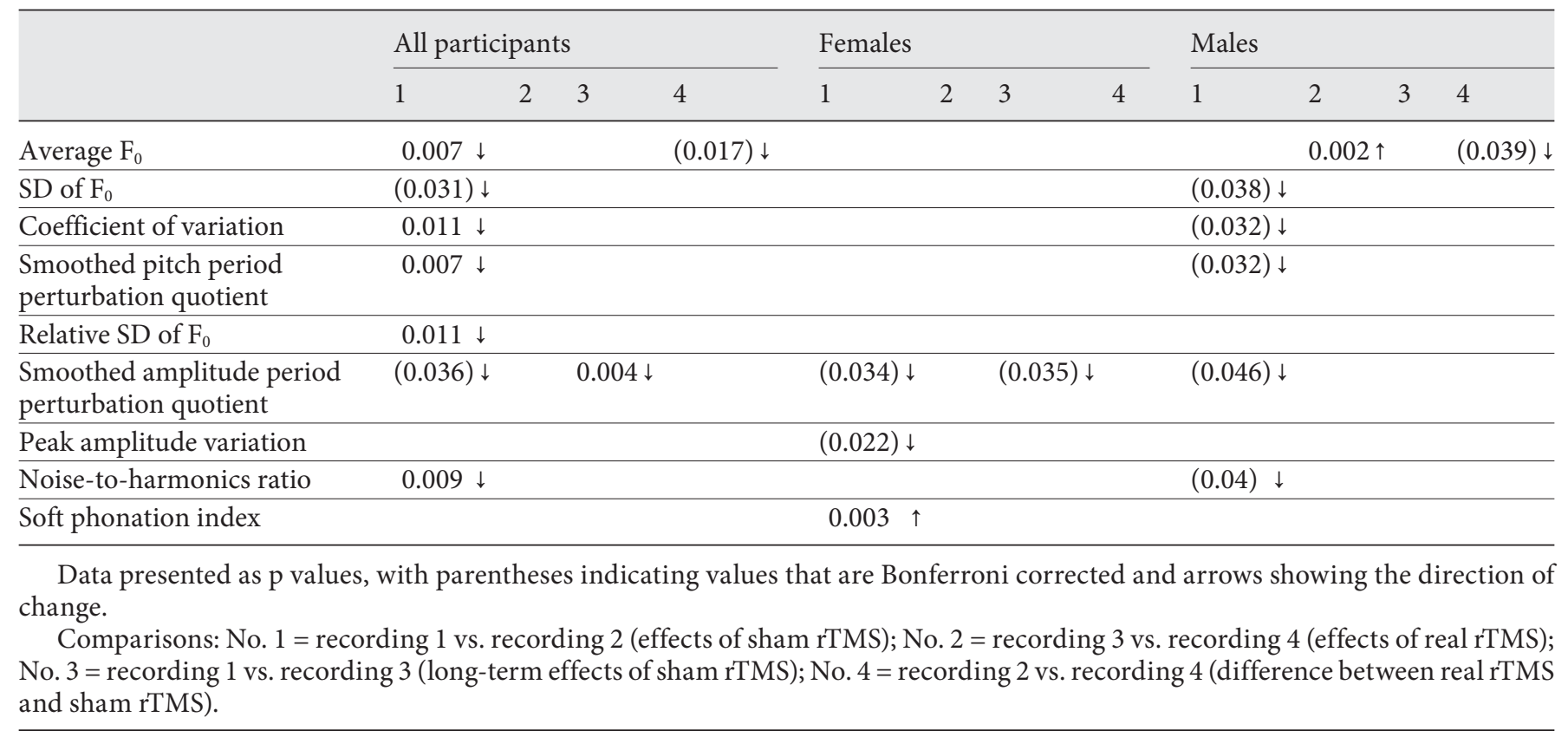

ences between recordings. In the entire group, there was a significant reduction $(\mathrm{p} \leq 0.0125)$ in $\mathrm{F}_{0}$, coefficient of variation, smoothed pitch period perturbation quotient, variability in $\mathrm{F}_{0}$ and noise-to-harmonics ratio (5 of the selected 9 relevant parameters), i.e. $\mathrm{F}_{0}$ decreased as did the variability in $\mathrm{F}_{0}$ and the amount of jitter (smoothed amplitude period perturbation quotient) and noise-toharmonics ratio as an effect of sham rTMS. The only oth- er significant effects found in females and males were an increase in soft phonation index in females as a result of sham rTMS and an increase in $\mathrm{F}_{0}$ in males as a result of real rTMS.

Consequently, on a group level, the only significant result seemed to be the effect of sham rTMS (i.e. the placebo effect). 


\section{Discussion}

In summary, rTMS did not seem to affect the speech and voice of the 10 participants with PD included in the present study. There are a number of possible reasons for this. The first reason concerns patient selection. These individuals did not have any severe symptoms of the disease in general, nor did they have any pronounced problems with speech or voice. Maximum fricative duration varied between the 4 recordings between 14.5 and $16.2 \mathrm{~s}$, and maximum phonation time varied between 15.2 and $17.5 \mathrm{~s}$. This is very close to the performance of 59 control subjects [18], who showed a maximum fricative duration of $19.3 \pm 11.8 \mathrm{~s}(\mathrm{SD})$ and a maximum phonation time of $15.5 \pm 6.2 \mathrm{~s}$. The performance of a group of individuals with PD included in the same study [18] was maximum fricative duration $11.5 \pm 6.3 \mathrm{~s}$ and maximum phonation time $15.4 \pm 8.3 \mathrm{~s}$. Diadochokinetic rates, however, were slightly below the performance of healthy subjects, mean diadochokinetic rate varied between 5.3 and 5.4 syllables per second, which is to be compared to a mean of $6.8 \pm$ $1.1 \mathrm{~s}$ in the 59 control subjects referred to previously. Sentence intelligibility was also $>90 \%$ in all participants, with a group mean of $97-99 \%$. Consequently, measures of speech touched on the performance of healthy subjects, and did not give room for extensive improvements.

The more sensitive acoustic measures revealed no effects of rTMS beyond the significant effects of placebo. Five of the nine selected MDVP parameters were improved after sham stimulation, the improvement being mainly accounted for by the male participants. In an investigation using MDVP to measure voice samples in various populations, including $\mathrm{PD}$, the most abnormal parameters were reported to be peak-amplitude variation, fundamental frequency variation, and short- or long-term variability of the peak-to-peak amplitude (sAPQ) [19]. Short-term cycle-tocycle variations such as jitter and shimmer are also often reported to be increased in individuals with PD [2]. These parameters were all decreased in the present study as an effect of sham rTMS, and SAPQ could also be interpreted as having a long-term placebo effect.

How can the placebo effect be explained? A few previous studies have also noted placebo effects as a consequence of treatment with rTMS. Of the 10 studies included in the meta-analysis of Elahi et al. [14], 2 studies reported effects of sham stimulation. One study evaluated high-frequency stimulation $(5 \mathrm{~Hz})$ of 10 patients [20] with a significant placebo effect, although smaller than the effects of real rTMS. The second study [21] investigated the effects of low-frequency stimulation $(0.2 \mathrm{~Hz})$ of 85 patients and found no differences between real and sham rTMS. A significant placebo effect was also noted in a study of the effects of rTMS on chronic tinnitus [22]. One explanation of the prevalence of placebo effects in these populations might be what is also known as the Hawthorne effect, the simple fact that a person is the object of experimental manipulation and in this case using new and advanced technical instruments is enough to create a treatment effect. It should be noted, however, that the placebo effect found in the acoustic voice parameters in the present study was not paralleled by any changes in UPDRS III. It is conceivable that the fact that speech and voice were included as part of the experimental protocol made the participants particularly aware of the possibility of therapy-induced speech and voice changes and created a placebo effect.

Another explanation of the lack of effect of rTMS on speech and voice in the present study concerns site of stimulation. The stimulation sites aiming to affect the motor symptoms in PD in previous studies have been frontal cortex, prefrontal cortex, dorsolateral prefrontal cortex and motor cortex. So far, only 1 study has focused specifically on the mouth area of the motor cortex [15] and this study reported effects on voice in both men and women. Obviously, to be able to explore the effects of rTMS on the phonatory and articulatory characteristics associated with dysarthria in PD, the stimulation sites need to be selected with that particular aim. In future studies, laryngeal, lip and tongue areas of the primary motor cortex should be targeted in order to evaluate possible effects on voice and speech.

Moreover, voice and speech measures need to be selected carefully to evaluate the effects of rTMS. The MDVP has potential to display small and/or incipient changes in phonation [17], but measures such as voice range and speech range profile, long-term phonatory stability, vowel space and the slope of formant 2 are also promising in terms of sensitivity to treatment effects $[7,8]$.

To conclude, although there were no significant effects of rTMS on speech and voice in the 10 participants with PD included in the present study, there were not any harmful effects, and thus it was concluded to be a safe treatment. Future research will include evaluating more severely affected individuals and also comparing rTMS stimulation with and without medication. In research on deep brain stimulation, the treatment effects on voice and speech have been found to be contrary to the effects on global motor function. It remains to be seen whether the beneficial effects on motor function found as a result of rTMS in studies of PD will be paralleled by improvements in speech and voice, given that more appropriate stimulation sites will be targeted. 


\section{References}

$>1$ Müller J, Wenning GK, Verny M, McKee A, Chaudhuri KR, Jellinger K, Poewe W, Litvan $\mathrm{I}$ : Progression of dysarthria and dysphagia in post-mortem-confirmed parkinsonian disorders. Arch Neurol 2001;58:259-264.

2 Adams S, Dykstra A: Hypokinetic dysarthria; in McNeil MR (ed): Clinical Management of Sensorimotor Speech Disorders. New York, Thieme, 2009, pp 166-186.

$>3$ Miller N, Allcock L, Jones D, Noble E, Hildreth AJ, Burn DJ: Prevalence and pattern of perceived intelligibility changes in Parkinson's disease. J Neurol Neurosurg Psychiatry 2007;78:1188-1190.

4 Kent RD: Perceptual sensorimotor speech examination for motor speech disorders; in McNeil MR (ed): Clinical Management of Sensorimotor Speech Disorders. New York, Thieme, 2009, pp 19-29.

5 Forrest K, Weismer G: Acoustic analysis of motor speech disorders; in McNeil MR (ed): Clinical Management of Sensorimotor Speech Disorders. New York, Thieme, 2009, pp 46-63.

$\checkmark 6$ Harel B, Cannizzaro M, Snyder PJ: Variability in fundamental frequency during speech in prodromal and incipient Parkinson's disease: a longitudinal case study. Brain Cogn 2004;56:24-29.

$>7$ Harel B, Cannizzaro M, Cohen H, Reilly N, Snyder PJ: Acoustic characteristics of Parkinsonian speech: potential biomarker of early disease progression and treatment. J Neurolinguistics 2004;17:439-453.
-8 Rosen KM, Kent RD, Delaney AL, Duffy JR: Parametric quantitative acoustic analysis of conversation produced by speakers with dysarthria and healthy speakers. J Speech Lang Hear Res 2006;49:395-411.

$\checkmark 9$ Ho AK, Bradshaw JL, Iansek R: For better or worse: the effect of levodopa on speech in Parkinson's disease. Mov Dis 2008;23:574580.

10 Ramig LO, Fox C, Sapir S: Speech treatment for Parkinson's disease. Expert Rev Neurother 2008;8:297-309.

11 Benabid AL, Chabardes S, Mitrofanis J, Pollak P: Deep brain stimulation of the subthalamic nucleus for the treatment of Parkinson's disease. Lancet Neurol 2009;8:67-81.

12 Klostermann F, Ehlen F, Vesper J, Nubel K, Gross M, Marzinzik F, Curio G, Sappok T: Effects of subthalamic deep brain stimulation on dysarthrophonia in Parkinson's disease. J Neurol Neurosurg Psychiatry 2008; 79:522-529.

13 Barker AT, Jalinous R, Freeston IL: Noninvasive magnetic stimulation of human motor cortex. Lancet 1985;2:1106-1107.

14 Elahi B, Elahi B, Chen R: Effect of transcranial magnetic stimulation on Parkinson motor function - systematic review of controlled clinical trials. Mov Dis 2009;24: 357-363.

15 Dias AE, Barbosa ER, Coracini K, Maia F, Marcolin MA, Fregni F: Effects of repetitive transcranial magnetic stimulation on voice and speech in Parkinson's disease. Acta Neurol Scand 2006;113:92-99.

16 Lillvik M, Allemark E, Karlström P, Hartelius L: Intelligibility of dysarthric speech in words and sentences: development of a computerised assessment procedure in Swedish. Logoped Phoniatr Vocol 1999;24:107-119.
17 Kent RD, Vorperian HK, Kent JF, Duffy RJ: Voice dysfunction in dysarthria: application of the Multi-Dimensional Voice Program. J Commun Dis 2003;36:281-306.

18 Hartelius L, Svensson P, Bubach A: Clinical assessment of dysarthria: performance on a dysarthria test by normal adult subjects, and by individuals with Parkinson's disease or with multiple sclerosis. Logoped Phoniatr Vocol 1993; 18:131-141.

19 Kent RD, Vorperian HK, Duffy JR: Reliability of the Multi-Dimensional Voice Program for the analysis of voice samples of subjects with dysarthria. Am J Speech Lang Pathol 1999;8:129-136

20 Siebner HR, Rossmeier C, Mentschel C, Peinemann A, Conrad B: Short-term motor improvement after sub-threshold $5-\mathrm{Hz}$ repetitive transcranial magnetic stimulation of the primary motor hand area in Parkinson's disease. J Neurol Sci 2000;178:91-94.

-21 Okabe S, Ugawa Y, Kanazawa I: The effectiveness of rTMS on Parkinson's Disease Study Group: $0.2-\mathrm{Hz}$ repetitive transcranial magnetic stimulation has no add-on effects as compared to a realistic sham stimulation in Parkinson's disease. Mov Dis 2003;18: 382-388.

22 Folmer RL, Carroll JR, Rahim A, Shi Y, Hal Martin W: Effects of repetitive transcranial magnetic stimulation (rTMS) on chronic tinnitus. Acta Otolaryngol Suppl 2006;556:96101. 